\title{
ARTICLE
}

\section{Ticagrelor inhibits the NLRP3 inflammasome to protect against inflammatory disease independent of the $\mathrm{P} 2 \mathrm{Y}_{12}$ signaling pathway}

\author{
Bo Huang ${ }^{1}$, Yufeng Qian ${ }^{2}$, Shujun Xie ${ }^{1,3}$, Xianhua Ye ${ }^{2}$, Hanwen Chen (iD) ${ }^{1}$ Zhifeng Chen ${ }^{1}$, Lihuan Zhang ${ }^{1}$, Jinming Xu ${ }^{4}$, Hu Hu ${ }^{5}$, \\ Shenglin $\mathrm{Ma}^{6}$, Paul Héroux ${ }^{7}$, Di Wang ${ }^{8}$, Han-Ming Shen ${ }^{9}$, Yihua $\mathrm{Wu}^{1}$ and Dajing $\mathrm{Xia}^{1}$
}

Ticagrelor is the first reversibly binding oral $\mathrm{P}_{2} \mathrm{Y}_{12}$ receptor antagonist to inhibit platelet activation and has been approved by the Food and Drug Administration for the treatment of coronary artery disease. At present, the other pharmacological functions of ticagrelor remain poorly understood. The NOD-, LRR-, and pyrin domain-containing protein 3 (NLRP3) inflammasome plays a critical role in the innate immune system, but its excessive activation also contributes to the pathogenesis of complex diseases. In this study, we systematically examined the effects of ticagrelor on the NLRP3 inflammasome and found that ticagrelor inhibits NLRP3 inflammasome activation in macrophages independent of its classic inhibitory effect on the $\mathrm{P}_{2} \mathrm{Y}_{12}$ signaling pathway. Further mechanistic studies demonstrate that ticagrelor attenuates the oligomerization of apoptosis-associated speck-like protein containing a CARD (ASC) by blocking chloride efflux, an effect achieved through the degradation of chloride intracellular channel proteins (CLICs) and blockade of the translocation of CLICs to the plasma membrane. Moreover, experiments on lipopolysaccharideinduced sepsis and alum-induced peritonitis in mice confirmed that ticagrelor mitigates the severity of systemic inflammation independent of $\mathrm{P}_{2} \mathrm{Y}_{12}$ receptor antagonism. Importantly, oral administration of ticagrelor rapidly and strongly inhibited NLRP3 inflammasome activation in peripheral blood mononuclear cells from patients with acute coronary syndrome. Overall, our study reveals a novel pharmacological function of ticagrelor in addition to its classic antiplatelet properties, which suggests that ticagrelor may serve as a potential therapeutic agent for use in NLRP3-associated diseases.

Keywords: Ticagrelor; NLRP3 inflammasome; P2Y 12 ; Chloride efflux; ASC

Cellular \& Molecular Immunology (2021) 18:1278-1289; https://doi.org/10.1038/s41423-020-0444-5

\section{INTRODUCTION}

Ticagrelor is the first reversibly binding oral $\mathrm{P}_{2} \mathrm{Y}_{12}$ receptor antagonist to inhibit platelet activation in patients with coronary artery disease $(C A D)^{1}$ and was approved by the US Food and Drug Administration in 2011. ${ }^{2}$ Compared to clopidogrel, a frequently used $\mathrm{P}_{2} \mathrm{Y}_{12}$ receptor inhibitor, ticagrelor has better therapeutic effects in preventing vascular events and death in patients with acute coronary syndrome (ACS), based on the Study of Platelet Inhibition and Patient Outcomes. ${ }^{3}$ At present, ticagrelor is widely preferred over numerous antiplatelet agents, such as clopidogrel, in patients and recommended as a first-line antiplatelet treatment in clinical guidelines, ${ }^{3-6}$ but other possible pharmacological functions of ticagrelor remain poorly documented.
In addition to its classic antiplatelet activity mediated by inhibition of the $\mathrm{P}_{2} \mathrm{Y}_{12}$ signaling pathway, there is accumulating evidence suggesting ticagrelor has other pharmacological functions. For instance, it was reported that compared to clopidogrel, ticagrelor contributes to lower mortality from inflammation-associated diseases such as sepsis and infection. ${ }^{7,8}$ A clinical trial further revealed that ticagrelor was capable of reducing interleukin-6 (IL-6) and tumor necrosis factor-a (TNF-a) levels in diabetic patients with nonST segment elevation ACS. ${ }^{9}$ Ticagrelor combined with rosuvastatin decreased myocardial mRNA levels of IL-1 $\beta$, IL-6, and NOD-, LRR-, and pyrin domain-containing protein 3 (NLRP3) in diabetic rats suffering from ischemia-reperfusion injury. ${ }^{10}$ Thus, the abovementioned findings indicate that ticagrelor may regulate inflammation, but little is known about the underlying mechanisms.

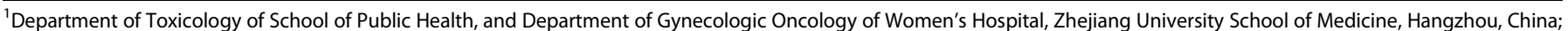

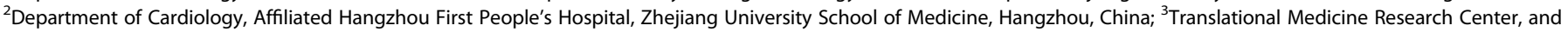

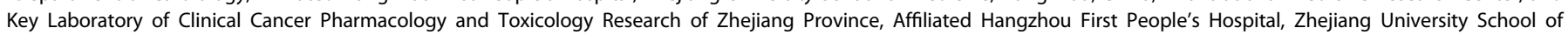

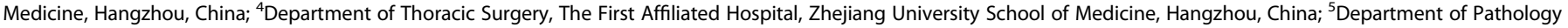

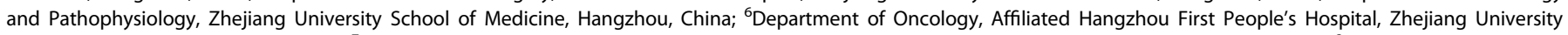

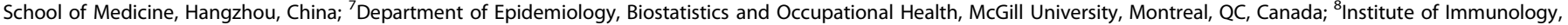

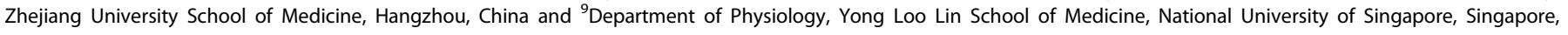
Singapore

Correspondence: Yihua Wu (georgewu@zju.edu.cn) or Dajing Xia (dxia@zju.edu.cn)

These authors contributed equally: Bo Huang, Yufeng Qian, Shujun Xie.
}

Received: 6 October 2019 Accepted: 12 April 2020

Published online: 10 June 2020 
The NLRP3 inflammasome provides a protective immune response against harmful stimuli. ${ }^{11-13}$ Upon activation, NLRP3 functions as a pattern recognition receptor in the cytosol by sensing pathogen- or danger-associated molecular patterns. It then oligomerizes to create a scaffold to recruit the adaptor protein apoptosis-associated specklike protein containing a CARD (ASC). It is crucial that ASC nucleates to generate long filaments for inflammasome activation. ASC in turn interacts with pro-caspase- 1 , mediating its maturation into active caspase-1. This results in caspase-1-dependent activation and release of IL-1 $\beta$ and IL-18, which induce a type of inflammatory cell death, pyroptosis. ${ }^{11,12,14,15}$ To date, inappropriate activation of the NLRP3 inflammasome has been implicated in a variety of human diseases, such as atherosclerosis, acute myocardial infarction, and type 2 diabetes. ${ }^{11,16,17}$ NLRP3 inflammasome blockade plays a protective role in inflammatory diseases. ${ }^{18,19}$ Therefore, this particular inflammasome appears to be a legitimate target for the development of therapeutic agents. At present, some small-molecule inhibitors, such as MCC950 and CY-09, and endogenous molecules, such as dopamine and bile acids, have been reported to inhibit the activation of the NLRP3 inflammasome. ${ }^{19-22}$ However, these inhibitors are mainly at the preclinical stage and are still far from actual clinical application. Therefore, the possible inhibition of NLRP3 inflammasome activation by the anticoagulant ticagrelor became the subject of this investigation.

In this study, we report a novel pharmacological activity of ticagrelor in innate immune responses in addition to its classic antiplatelet properties. We show that ticagrelor functions as a potent NLRP3 inhibitor in macrophages in vitro and in NLRP3dependent mouse models in vivo, as well as in ACS patients. Our work thus supports developing ticagrelor as a potential therapeutic agent for NLRP3-associated diseases.

\section{RESULTS}

Ticagrelor inhibits the activation of the NLRP3 inflammasome To examine the potential anti-inflammatory activity of ticagrelor, we stimulated lipopolysaccharide (LPS)-primed mouse bone marrow-derived macrophages (BMDMs) with nigericin to activate the NLRP3 inflammasome. Ticagrelor treatment (10-20 $\mu \mathrm{M})$ before or after LPS stimulation significantly inhibited both caspase-1 maturation and IL-1 $\beta$ secretion in a dose-dependent manner (Fig. 1a, b and Supplemental Fig. 1a). However, in contrast to treatment after LPS stimulation (Fig. 1a, c, lanes 7-12), ticagrelor treatment before LPS stimulation (Fig. 1a, c, lanes 1-6) was a

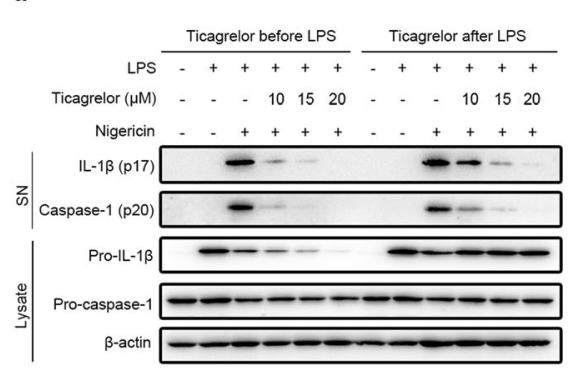

b

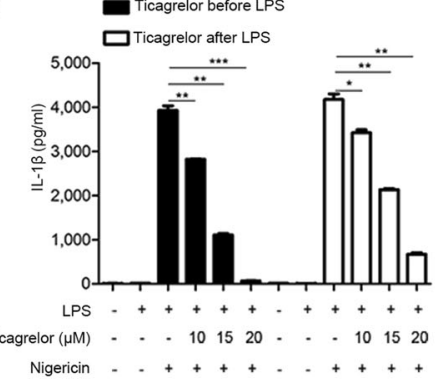

d

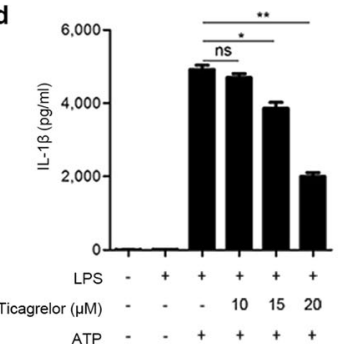

f

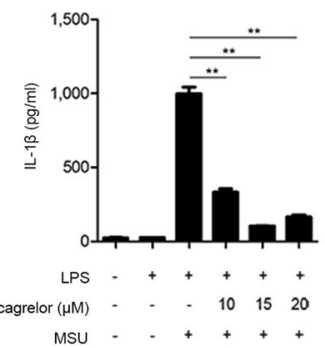

c
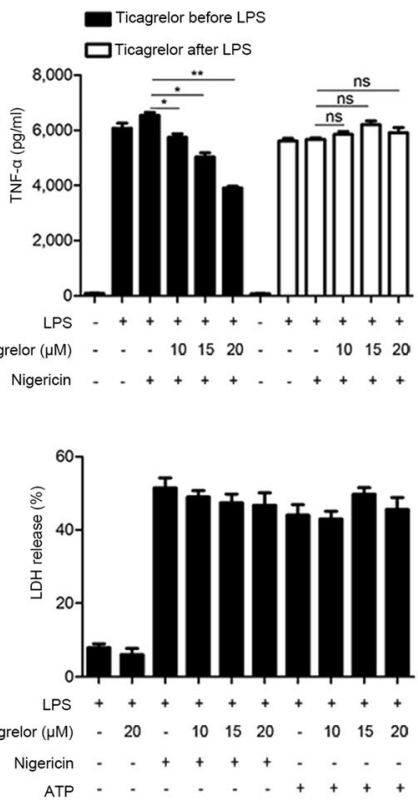

h

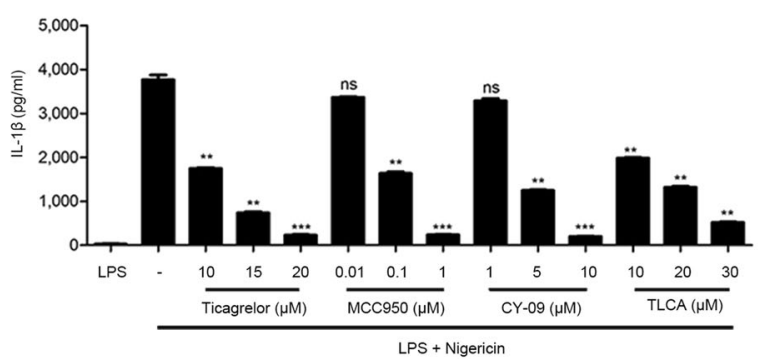

Fig. 1 Ticagrelor inhibits the activation of the NLRP3 inflammasome in macrophages. a-c BMDMs were treated with different doses of ticagrelor $(10-20 \mu \mathrm{M})$ for $30 \mathrm{~min}$ before $5.5 \mathrm{~h}$ of LPS priming and then stimulated with nigericin for $30 \mathrm{~min}$, or BMDMs were primed with LPS for $6 \mathrm{~h}$ and then treated with ticagrelor $(10-20 \mu \mathrm{M})$ and nigericin simultaneously for $30 \mathrm{~min}$. Supernatants (SN) and cell lysates (Lysate) were analyzed by immunoblotting (a), and supernatants were analyzed by enzyme-linked immunosorbent assay (ELISA) to determine IL-1 $\beta$ (b) and TNF- $\alpha$ levels (c). d-f LPS-primed BMDMs were treated with ticagrelor $(20 \mu \mathrm{M})$ and then immediately stimulated with ATP (d), alum (e), or MSU (f). Supernatants were analyzed by ELISA to determine IL-1 $\beta$ levels. $\mathbf{g}$ The release of LDH into supernatants by LPS-primed BMDMs treated with ticagrelor ( $20 \mu \mathrm{M})$ and then stimulated with ATP or nigericin was measured. h LPS-primed BMDMs were treated with different doses of ticagrelor, MCC950, CY-09, or TLCA and then stimulated with nigericin; supernatants were analyzed by ELISA to determine IL-1 $\beta$ levels. i LPS-primed BMDMs were treated with ticagrelor, MCC950, CY-09, or TLCA for different times as indicated and then stimulated with nigericin; supernatants were analyzed by ELISA to determine IL-1 $\beta$ levels. Data are presented as the mean \pm SEM; ${ }^{*} P<0.05$, ${ }^{* *} P<0.01,{ }^{* * *} P<0.001$, ns: $P>0.05$; unpaired, two-tailed $t$-test 
a
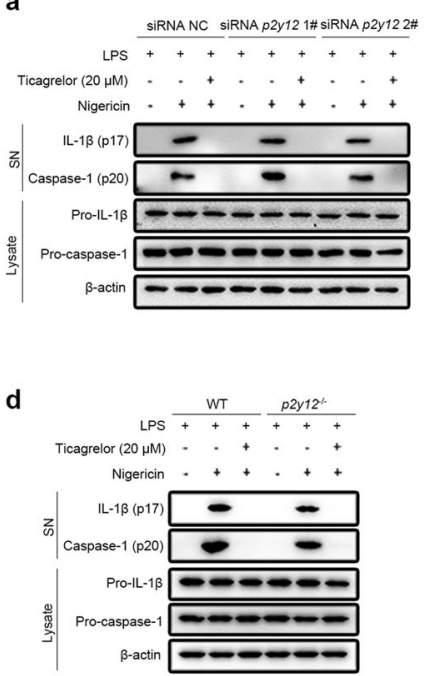

g

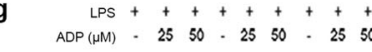

Ticagrelor $(20 \mu \mathrm{M})$ - . . . . . +++

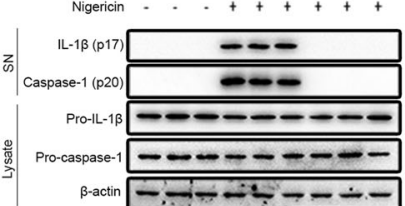

h

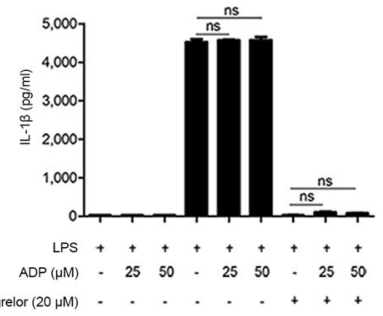

m

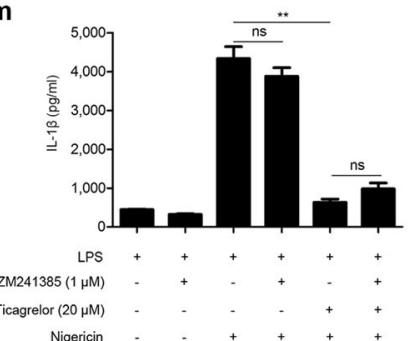

b

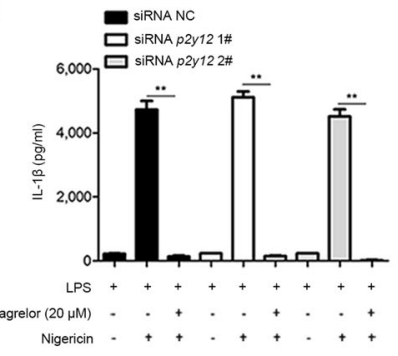

e

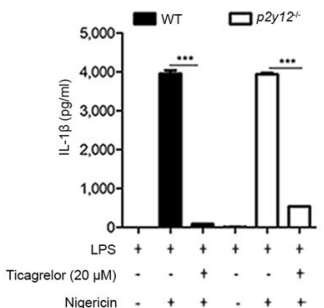

i

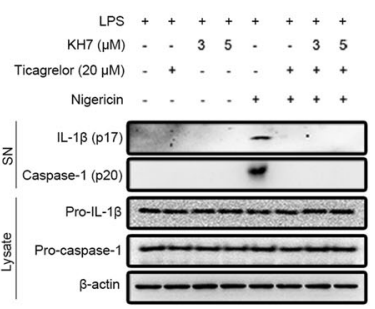

j
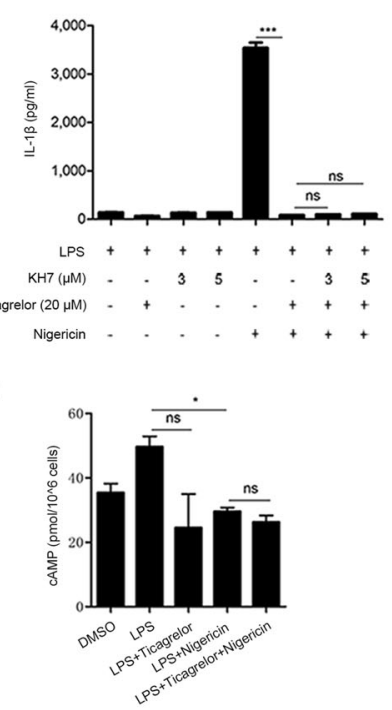
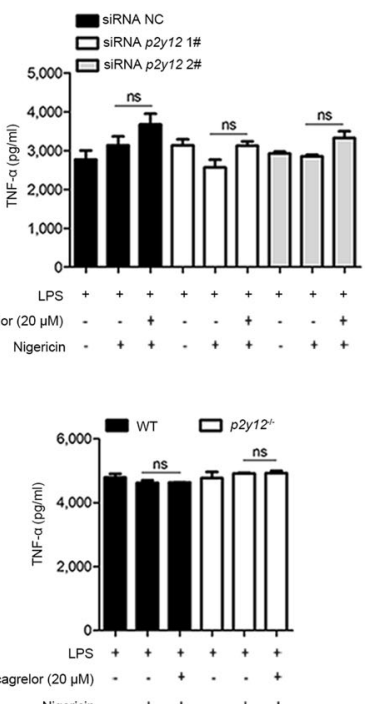

k

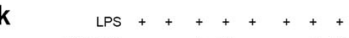

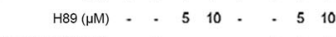

Ticagrelor $(20 \mu \mathrm{M}) \ldots+\ldots+++$

Nigericin - . . + + + +
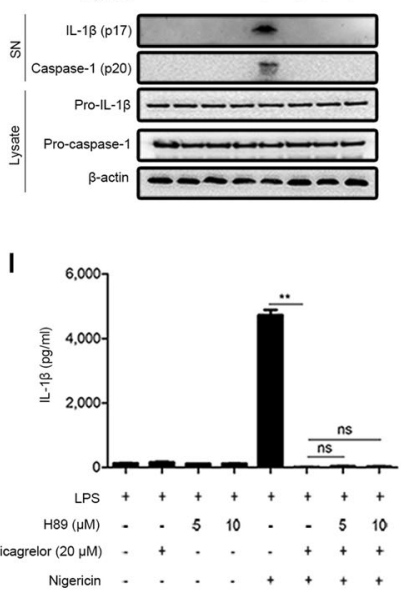

Fig. 2 Ticagrelor controls the NLRP3 inflammasome independent of the P2Y 12 signaling pathway. a-c LPS-primed BMDMs were treated with p2y12-specific siRNA before ticagrelor $(20 \mu \mathrm{M})$ treatment and then stimulated with nigericin. Supernatants (SN) and cell lysates (Lysate) were analyzed by immunoblotting (a), and supernatants were analyzed by ELISA to determine IL-1 $\beta$ (b) and TNF- $\alpha$ levels (c). $\mathbf{d}-\mathbf{f}$ LPS-primed BMDMs from wild-type or $p 2 y 12^{-/-}$mice were treated with ticagrelor $(20 \mu \mathrm{M})$ and then stimulated with nigericin. Supernatants (SN) and cell lysates (Lysate) were analyzed by immunoblotting (d), and supernatants were analyzed by ELISA to determine IL-1 $\beta$ (e) and TNF- $\alpha$ levels (f). $\mathbf{g}$, $\mathbf{h}$ LPSprimed BMDMs were treated with different doses of ADP for 5 min before ticagrelor $(20 \mu \mathrm{M})$ treatment and then stimulated with nigericin; supernatants (SN) and cell lysates (Lysate) were analyzed by immunoblotting (g), and supernatants were analyzed by ELISA to determine IL-1 $\beta$ levels (h). $\mathbf{i}$, $\mathbf{j}$ LPS-primed BMDMs were treated with different doses of KH7 for 30 min before ticagrelor $(20 \mu \mathrm{M})$ treatment and then stimulated with nigericin; supernatants (SN) and cell lysates (Lysate) were analyzed by immunoblotting (i), and supernatants were analyzed by ELISA to determine IL-1 $\beta$ levels $(\mathbf{j})$. k, I LPS-primed BMDMs were treated with different doses of H89 for 30 min before ticagrelor (20 $\mu \mathrm{M})$ treatment and then stimulated with nigericin; supernatants (SN) and cell lysates (Lysate) were analyzed by immunoblotting (k), and supernatants were analyzed by ELISA to determine IL-1 $\beta$ levels (I). $\mathbf{m}$ LPS-primed BMDMs were treated with ZM241385 (1 $\mu \mathrm{M})$ for 30 min before ticagrelor (20 $\mu \mathrm{M})$ treatment and then stimulated with nigericin, and supernatants were analyzed by ELISA to determine IL-1 $\beta$ levels. $\mathbf{n}$ The level of cAMP induction in LPS-primed BMDMs treated with ticagrelor $(20 \mu \mathrm{M})$ and then stimulated with nigericin was measured. Data are presented as the mean \pm SEM; ${ }^{*} P<0.05,{ }^{* *} P<0.01,{ }^{* * *} P<0.001, \mathrm{~ns}: P>0.05$; unpaired, two-tailed $t$-test 
effective in decreasing LPS-induced pro-IL-1 $\beta$ expression and TNFa production. Consistently, ticagrelor treatment before LPS stimulation but not after LPS stimulation impaired LPS-induced NLRP3 expression (Supplemental Fig. 1b). In addition, the relative mRNA levels of II1 $\beta$, Tnfa, NIrp3, Asc, and Caspase-1 were not affected by ticagrelor treatment after LPS stimulation (Supplemental Fig. $1 \mathrm{c}-\mathrm{g}$ ). Therefore, whether ticagrelor affects the priming stage is dependent on whether it is used before or after LPS stimulation. We thus continued our investigation with the assumption that the priming stage was not affected by ticagrelor treatment after LPS stimulation. Consistently, we also found that ticagrelor had similar effects on NLRP3 inflammasome activation in mouse peritoneal macrophages (PMs) (Supplemental Fig. 2a-d). To exclude the possibility that ticagrelor inhibits NLRP3 inflammasome activation in macrophages through platelet contamination, we performed experiments on mouse platelets. However, neither IL-1 $\beta$, caspase- 1 , and TNF-a secretion into supernatants nor NLRP3, ASC, pro-caspase- 1 , and pro-IL-1 $\beta$ expression in cell lysates was detected when platelets were stimulated with LPS and nigericin (Supplemental Fig. 2e-g). Apart from nigericin, aluminum salts (alum), ATP, and monosodium urate crystals (MSU) can also trigger NLRP3 inflammasome activation. ${ }^{23,24}$ Consistently, ticagrelor inhibited caspase- 1 activation and IL-1 $\beta$ cleavage induced by different NLRP3 inflammasome stimuli in a dose-dependent manner (Fig. 1d-f). We also found that the ticagrelor-induced inhibition of the NLRP3 inflammasome was not due to blockade of pyroptosis, as cell viability measured as lactate dehydrogenase release was not affected by ticagrelor (Fig. 1g). Other intracellular pattern recognition receptors, such as absent in melanoma 2 (AIM2) and NLR family, CARD domain-containing protein 4 (NLRC4), also form inflammasomes. ${ }^{24-26}$ We further examined the anti-inflammatory action of ticagrelor and found that it inhibited the poly (dA:dT)-induced activation of the AIM2 inflammasome (Supplemental Fig. 3a, b) but had no effect on the Salmonella typhimurium-induced activation of the NLRC4 inflammasome (Supplemental Fig. 3c, d). We next compared the inhibitory effect of ticagrelor on the NLRP3 inflammasome with the effects of other known inhibitors and found that the inhibitory activity of ticagrelor against the NLRP3 inflammasome was comparable to that of MCC950, CY-09, and taurolithocholic acid (TLCA) (Fig. 1h, i). Thus, these results indicate that ticagrelor potently and broadly inhibits NLRP3 inflammasome activation in macrophages.

Ticagrelor inhibits the NLRP3 inflammasome independent of the $\mathrm{P} \mathrm{Y}_{12}$ signaling pathway

We next determined whether the $P 2 Y_{12}$ signaling pathway is involved in the ticagrelor-induced inhibition of the NLRP3 inflammasome in macrophages. Consistent with a previous report, ${ }^{27}$ we found that LPS exposure significantly downregulated the expression of $\mathrm{P}_{2} \mathrm{Y}_{12}$ in macrophages (Supplemental Fig. 4a, b). To validate the above findings, we used two genetic approaches to silence $\mathrm{P}_{2} \mathrm{Y}_{12}$ and then examined the effects of ticagrelor. First, BMDMs were transfected with small interfering RNA to knockdown P2y12 (Supplemental Fig. 4c). Interestingly, ticagrelor retained its inhibitory effect on the activation of the NLRP3 inflammasome in P2y12-knockdown cells (Fig. 2a-c). Second, we utilized $p 2 y 12^{-/-}$BMDMs obtained from $p 2 y 12^{-/-}$mice (Supplemental Fig. $4 d)^{28}$ and found that ticagrelor was still effective in inhibiting caspase- 1 maturation and IL-1 $\beta$ secretion in these cells (Fig. 2d-f). Next, we further tested whether manipulation of the $\mathrm{P}_{2} \mathrm{Y}_{12}$ signaling pathway affects ticagrelor activity. ADP binds to $\mathrm{P}_{2} \mathrm{Y}_{12}$ to induce platelet activation, ${ }^{29}$ and ticagrelor binds reversibly to the receptor at a different site to inhibit ADP signaling. ${ }^{1}$ Notably, our results showed that the addition of ADP did not affect the activation of the NLRP3 inflammasome in macrophages or change the ticagrelor-induced inhibition of the
NLRP3 inflammasome (Fig. 2g, h). As a Gi-coupled receptor, the $\mathrm{P}_{2} \mathrm{Y}_{12}$ receptor inhibited by ticagrelor activates adenylate cyclase, which promotes the production of cyclic AMP (cAMP) in platelets. ${ }^{1,30,31}$ However, the ticagrelor-induced inhibition of the NLRP3 inflammasome in macrophages was not affected by the adenylate cyclase inhibitor $\mathrm{KH} 7$ (Fig. $2 \mathrm{i}$, j). The role of the CAMPPKA pathway, which is downstream of $\mathrm{P}_{2} \mathrm{Y}_{12}$ signaling, was also excluded by experiments showing that the PKA inhibitor H89 had no effect on the ticagrelor-induced inhibition of the NLRP3 inflammasome (Fig. 2k, I). Recently, ticagrelor has been reported to increase extracellular adenosine levels via blockade of equilibrative nucleoside transporter 1 on platelets, which activates the $A_{2 A}$ adenosine receptor, leading to accumulation of CAMP. ${ }^{32}$ Notably, CAMP disrupts inflammasome assembly by binding directly to NLRP3. ${ }^{33}$ It is thus important to determine the possible involvement of adenosine and CAMP in ticagrelor-induced inhibition of the NLRP3 inflammasome. In this study, a specific inhibitor of the $A_{2 A}$ adenosine receptor, ZM241385, did not affect the inhibitory effect of ticagrelor on the NLRP3 inflammasome (Fig. $2 \mathrm{~m}$ ). We also measured CAMP levels and found that CAMP production in BMDMs was not increased by ticagrelor (Fig. 2n). Thus, we provide multiple lines of evidence showing that ticagrelor inhibits the activation of the NLRP3 inflammasome independent of the P2Y 12 signaling pathway in macrophages.

Ticagrelor specifically attenuates ASC complex formation to inhibit the NLRP3 inflammasome

Next, we sought to investigate the mechanisms by which ticagrelor inhibits the NLRP3 inflammasome. It has been reported that NLRP3 expression induced by nuclear factor $\mathrm{KB}$ signaling in LPS-primed macrophages is not sufficient for inflammasomedependent IL-1 $\beta$ secretion, ${ }^{34}$ while deubiquitination of NLRP3 is critical for inflammasome assembly and activation. ${ }^{35,36}$ In this study, we found that ticagrelor did not induce higher levels of NLRP3 ubiquitination in macrophages than LPS priming alone (Supplemental Fig. 5a), suggesting that ticagrelor may affect the downstream posttranslational modification of NLRP3. Studies have shown that NLRP3 interacts with ASC through the pyrin domain to initiate inflammasome formation. ${ }^{37-39}$ Here, we treated BMDMs with ticagrelor for $0-30 \mathrm{~min}$ prior to nigericin stimulation and found that ticagrelor did not reduce the expression level of NLRP3 or ASC (Supplemental Fig. 5b, c). Next, coimmunoprecipitation analysis demonstrated that ticagrelor had no effect on the NLRP3-ASC interaction (Supplemental Fig. $5 \mathrm{~d}$, e). It is well known that homogeneous pyrin domain association promotes ASC filament formation, leading to NLRP3 inflammasome activation. ${ }^{37-39}$ Our results revealed that ticagrelor significantly reduced the formation of perinuclear structures called ASC-speck in macrophages primed with LPS and nigericin, with similar effects observed in both wild-type cells and $p 2 y 12^{-1-}$ cells (Fig. 3a, b). Consistently, ASC oligomerization triggered by nigericin was also markedly disturbed by ticagrelor in both wild-type cells and p2y $12^{-1-}$ cells (Fig. 3c). Thus, the abovementioned results clearly indicate that ticagrelor specifically targets the oligomerization of ASC to inhibit inflammasome activation without affecting the NLRP3-ASC interaction, a process likely to be independent of the $\mathrm{P}_{2} \mathrm{Y}_{12}$ receptor. It has been reported that inhibition of Syk or JNK abolishes ASC oligomerization without affecting the interaction of NLRP3 with ASC. ${ }^{40}$ We thus investigated whether Syk or JNK is implicated in the inhibitory effects of ticagrelor on the NLRP3 inflammasome. Interestingly, we found that ticagrelor-induced inhibition of the NLRP3 inflammasome was not affected in Sykknockdown BMDMs or JNK-knockout iBMDMs (Supplemental Fig. 6a-e). Given all the results described above, our studies clearly indicate that ticagrelor inhibits NLRP3 inflammasome activation via suppression of ASC oligomerization independent of the $\mathrm{P}_{2} \mathrm{Y}_{12}$ receptor. 
a

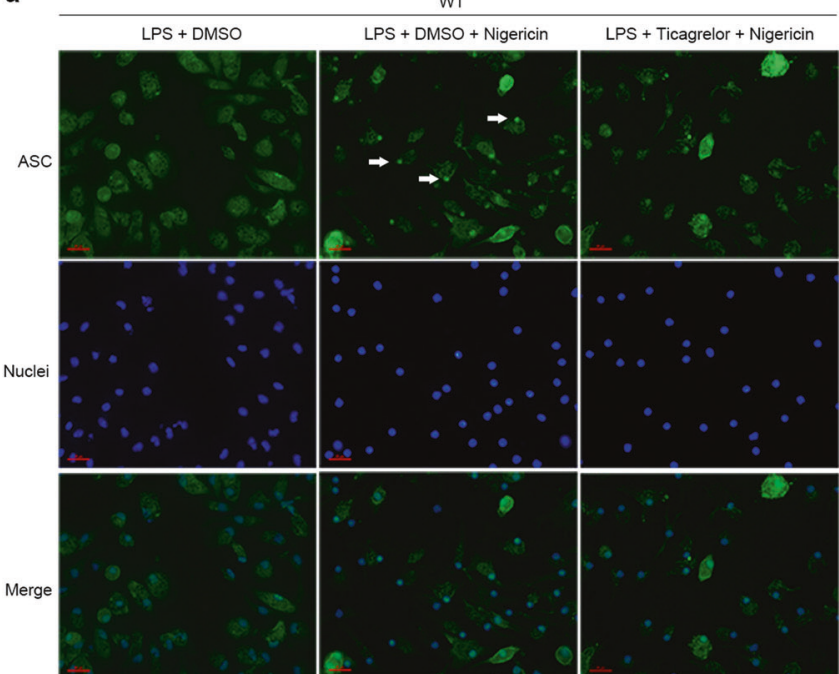

$p 2 y 12^{+}$

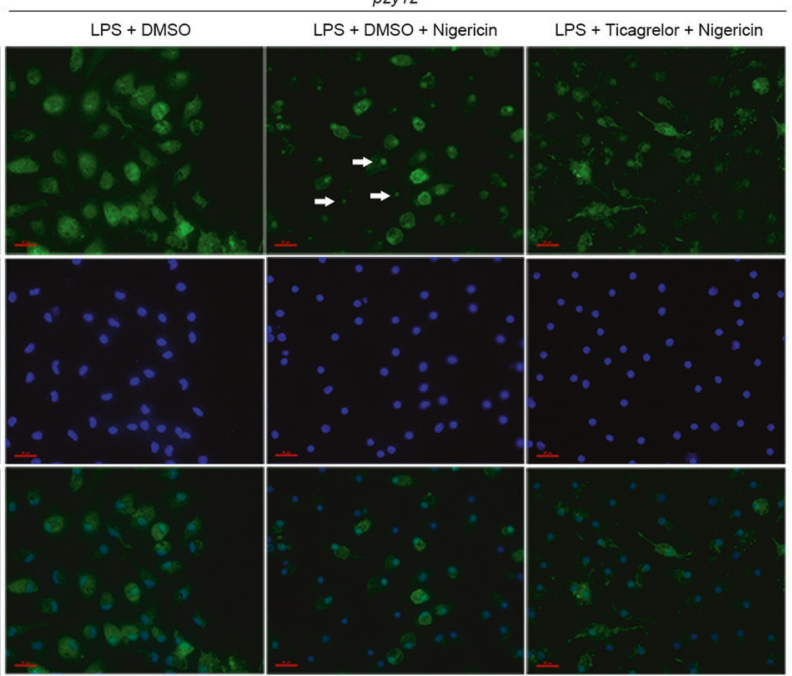

b

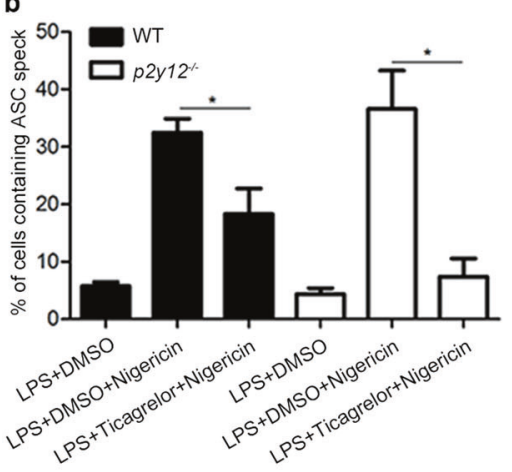

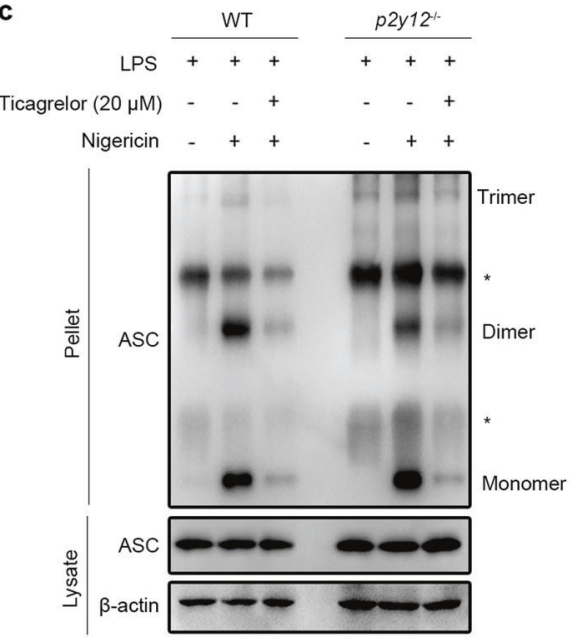

Fig. 3 Ticagrelor specifically attenuates ASC complex formation to inhibit the NLRP3 inflammasome. a Immunofluorescence images of ASCspeck formation (white arrows) in LPS-primed BMDMs (wild-type and $p 2 y 12^{-1-}$ ) treated with ticagrelor $(20 \mu \mathrm{M})$ and then stimulated with nigericin. Images were captured at an original magnification of $\times 40$; green, ASC; blue, nuclei. Scale bars, $50 \mu \mathrm{m}$. b Analysis of three independent experiments assessing ASC-speck formation to calculate the percentage of cells containing ASC-speck from data collected as in (a). c Immunoblots of ASC oligomerization in lysates of LPS-primed BMDMs (wild-type or $p 2 y 12^{-1-}$ ) treated with ticagrelor (20 $\mu$ M) and then stimulated with nigericin. * indicates a nonspecific band. Data are presented as the mean $\pm \mathrm{SEM} ;{ }^{*} P<0.05,{ }^{* *} P<0.01,{ }^{* * *} P<0.001, \mathrm{~ns}$ : $P>0.05$; unpaired, two-tailed $t$-test

Ticagrelor inhibits ASC oligomerization by blocking chloride efflux It has recently been shown that chloride efflux is an essential upstream event for NLRP3 inflammasome activation and an increased chloride ion concentration in the cytosol blocks NLRP3 inflammasome activation and impairs the maturation and release of IL-1 $\beta .{ }^{41,42}$ Furthermore, ASC oligomerization is also chloride efflux dependent. ${ }^{41,43}$ We then investigated whether ticagrelor inhibits ASC oligomerization by regulating chloride efflux by using $\mathrm{N}$-(Ethoxycarbonylmethyl)-6-methoxyquinolinium bromide (MQAE). As MQAE is a fluorescence probe for chloride ions, a higher intensity of MQAE fluorescence indicates a lower level of chloride ions and vice versa. ${ }^{44}$ As shown in Fig. $4 a$, treatment with LPS and nigericin markedly increased the fluorescence intensity of $M Q A E$, indicating a lower level of intracellular chloride ions due to enhanced chloride efflux, a process completely blocked by ticagrelor (Fig. 4a). Consistent with a previous report, ${ }^{20}$ our results also showed that MCC950 but not CY-09 blocked chloride efflux completely (Fig. 4a), which validated our method to examine intracellular chloride. It is well known that chloride intracellular channel proteins (CLICs) mediate chloride efflux by translocation to the plasma membrane. ${ }^{41}$ CLICs comprise six members; only Clic1, Clic4, and Clic5 are expressed in BMDMs, and LPS stimulation can upregulate the expression of Clic4 and Clic5. ${ }^{41,45}$ Interestingly, we found that ticagrelor induced the degradation of Clic1, Clic4, and Clic5 (Fig. 4b). Similar results were also obtained in p2y $12^{-1-}$ BMDMs (Supplemental Fig. 7a, b). We then asked whether the proteasome or autophagy mediates ticagrelorinduced CLIC degradation. Our results showed that the proteasome inhibitor MG132 could not suppress ticagrelor-induced CLIC degradation (Fig. 4C), while the autophagy/lysosome inhibitor bafilomycin A1 restored the protein levels of the CLICs (Fig. 4d). Interestingly, we observed that ticagrelor increased the levels of LC3-II (Fig. 4e), and compared to bafilomycin A1 alone, ticagrelor plus the lysosome inhibitor bafilomycin A1 increased LC3-II levels (Fig. 4f), indicating that ticagrelor induces autophagic flux in LPSprimed macrophages, which is responsible for CLIC degradation. Importantly, bafilomycin A1 was able to enhance the level of IL-1 $\beta$ secretion decreased by ticagrelor (Fig. $4 \mathrm{~g}$ ). These results indicate that ticagrelor inhibits ASC oligomerization by blocking chloride efflux; however, it was difficult to observe ASC oligomerization in 
a
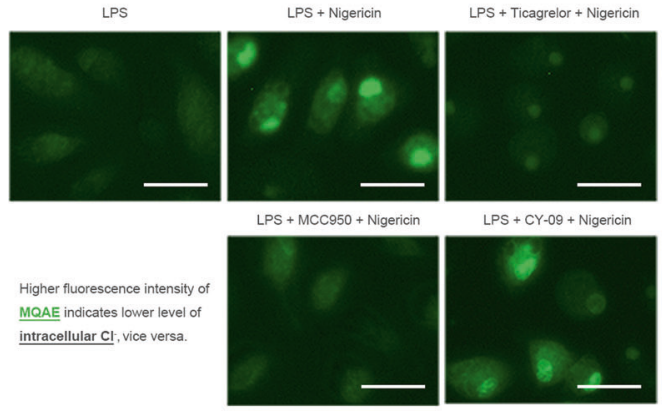

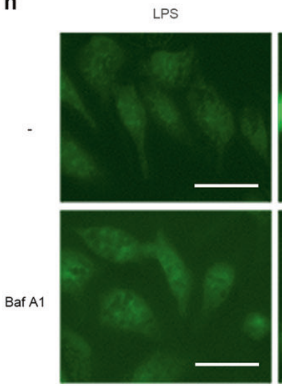

LPS + Nigericin

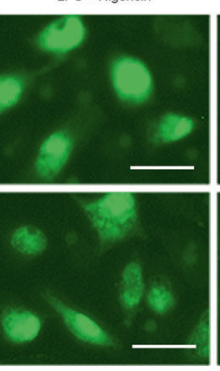

LPS + Ticagrelor + Nigericin

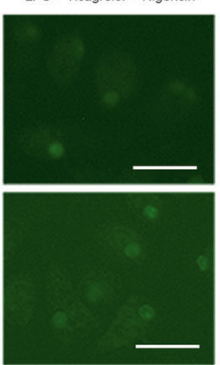

b

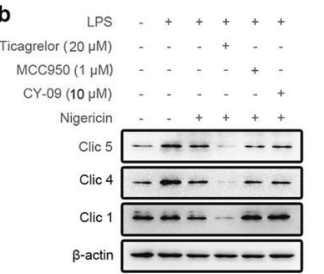

e

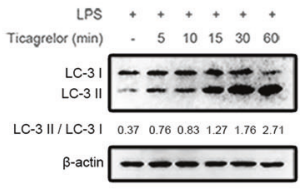

c

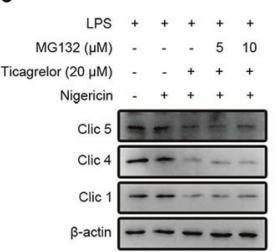

f

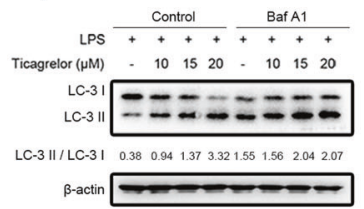

d

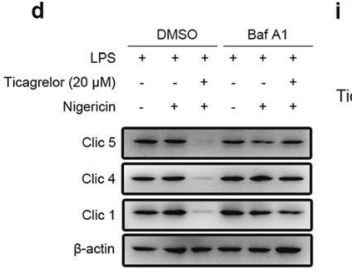

i g

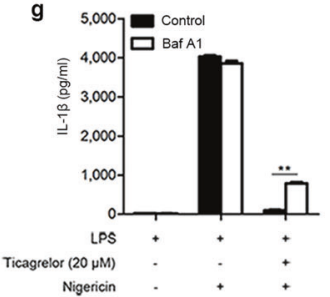

Ticagrelor $(20 \mu \mathrm{M})$

Nigericin

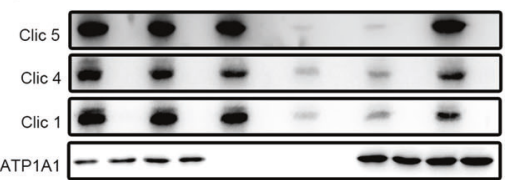

ATP1A1 - - -

GAPDH $-\infty-\infty-\infty-\infty-\cdots$

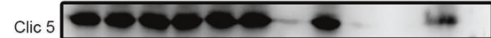

Clic $4-\infty-\infty-\infty-$

Clic 1

TP1A1

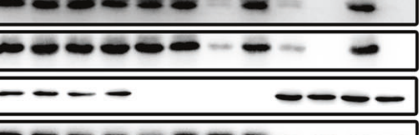

GAPDH $-\infty-\infty \ldots$

Fig. 4 Ticagrelor blocks chloride efflux to inhibit ASC oligomerization. a LPS-primed BMDMs were treated with ticagrelor (20 $\mu$ M), MCC950 $(1 \mu \mathrm{M})$, or CY-09 $(10 \mu \mathrm{M})$ before nigericin stimulation. BMDMs were then incubated with MQAE to detect intracellular chloride ions with fluorescence microscopy. Scale bars, $100 \mu \mathrm{m}$. b Immunoblots of Clic1, Clic4, and Clic5 in LPS-primed BMDMs treated with ticagrelor (20 $\mu$ M), MCC950 $(1 \mu \mathrm{M})$, or CY-09 $(10 \mu \mathrm{M})$ as indicated before nigericin stimulation are shown. c LPS-primed BMDMs were treated with different doses of MG132 for $2 \mathrm{~h}$ before ticagrelor $(20 \mu \mathrm{M})$ treatment and then stimulated with nigericin; cell lysates (Lysate) were analyzed by immunoblotting. d LPS-primed BMDMs were treated with $200 \mathrm{nM}$ bafilomycin A1 for $2 \mathrm{~h}$ before ticagrelor $(20 \mu \mathrm{M})$ treatment and then stimulated with nigericin; cell lysates (Lysate) were analyzed by immunoblotting. e LPS-primed BMDMs were treated with ticagrelor (20 $\mu$ M) for different times as indicated; cell lysates were analyzed by immunoblotting, and the ratio of LC-3 II/LC-3 I was evaluated with ImageJ. $\mathbf{f}$ LPSprimed BMDMs were incubated with $200 \mathrm{nM}$ bafilomycin A1 for $2 \mathrm{~h}$ before ticagrelor treatment; cell lysates were analyzed by immunoblotting, and the ratio of LC-3 II/LC-3 I was evaluated with ImageJ. g LPS-primed BMDMs were treated with $200 \mathrm{nM}$ bafilomycin A1 for $2 \mathrm{~h}$ before ticagrelor $(20 \mu \mathrm{M})$ treatment and then stimulated with nigericin; supernatants were analyzed by ELISA to determine IL-1 $\beta$ levels. $\mathbf{h}$ BMDMs treated as in $(\mathbf{g})$ were then incubated with MQAE to detect intracellular chloride ions with fluorescence microscopy. Scale bars, $100 \mu \mathrm{m}$. $\mathbf{i}$ The plasma membrane and cytosolic fractions of BMDMs treated as in $(\mathbf{g})$ were isolated to evaluate the translocation of CLICs by immunoblotting. Data are presented as the mean \pm SEM; ${ }^{*} P<0.05,{ }^{* *} P<0.01,{ }^{* * *} P<0.001$, ns: $P>0.05$; unpaired, two-tailed $t$-test.

bafilomycin A1-treated BMDMs, as bafilomycin A1 could not rescue $\mathrm{IL}-1 \beta$ secretion to a certain level. We further sought to investigate the underlying mechanisms. Intriguingly, ticagrelor still blocked chloride efflux in BMDMs treated with LPS and nigericin when bafilomycin A1 restored the protein levels of the CLICs (Fig. 4h). Under this condition, the NLRP3 agonist-induced translocation of cytosolic CLICs to the plasma membrane was suppressed by ticagrelor (Fig. 4i). Taken together, our results demonstrate that ticagrelor is capable of preventing CLIC plasma membrane translocation, thus blocking chloride efflux, increasing the intracellular chloride ion concentration, and subsequently leading to the inhibition of ASC oligomerization and eventually the suppression of NLRP3 inflammasome activation.

Ticagrelor mitigates the severity of inflammation in mouse models and patients with ACS

To explore the therapeutic potential of ticagrelor in NLRP3associated diseases, we established two animal models. First, we used a mouse model of alum-induced peritonitis, which is known to be mediated by the NLRP3 inflammasome. ${ }^{46}$ Remarkably, ticagrelor decreased the IL-1 $\beta$ concentration and numbers of peritoneal exudate cells (PECs) and neutrophils in the peritoneal lavage fluid of both wild-type mice and $p 2 y 12^{-1-}$ mice (Fig. 5a-c). Second, we established a mouse model of LPS-induced sepsis. It is known that intraperitoneal injection of LPS into mice elicits NLRP3-dependent IL-1 $\beta$ and IL-18 secretion in the serum. ${ }^{47}$ As expected, ticagrelor markedly reduced the serum levels of IL-1 $\beta$ and IL-18 but not the serum level of TNF-a (Fig. 5d-f). In agreement with our in vitro data, ticagrelor was found to have similar effects on LPS-induced sepsis in both wild-type mice and p $2 y 12^{-l-}$ mice (Fig. 5d-f). Furthermore, ticagrelor increased the survival of wild-type and $p 2 y 12^{-1-}$ mice injected with a lethal dose of LPS (Fig. $5 \mathrm{~g}$ ). Taken together, our results clearly demonstrate that ticagrelor mitigates the severity of systemic inflammation in mouse models independent of the $\mathrm{P}_{2} \mathrm{Y}_{12}$ receptor. Finally, we measured the anti-inflammatory effect of ticagrelor on human subjects via two approaches. First, we found that ticagrelor inhibited the activation of the NLRP3 inflammasome triggered by LPS and nigericin in peripheral blood mononuclear cells (PBMCs) from healthy volunteers (Supplemental Fig. $8 a-c$ ). It has been reported that oral ticagrelor treatment at a dosage of $90 \mathrm{mg}$ twice daily for 6-12 months prevents vascular events and death. ${ }^{3,48}$ Interestingly, at the same dose, our preliminary analysis showed that ticagrelor inhibited IL-1 $\beta$ secretion by PBMCs from patients 
a

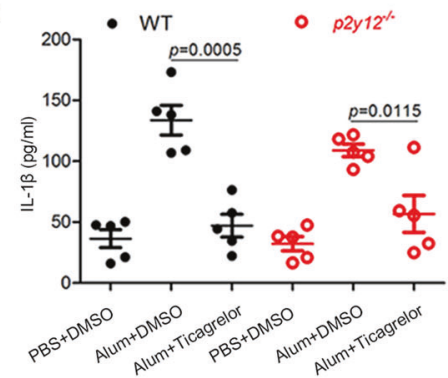

d

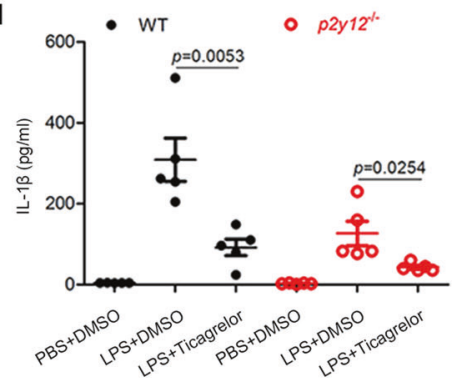

b

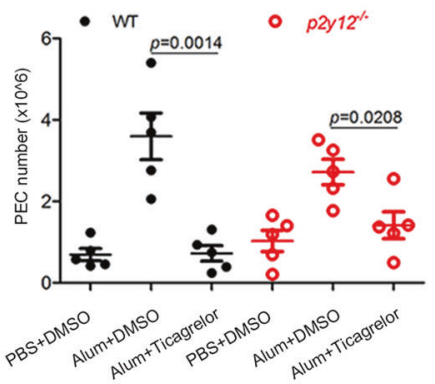

e

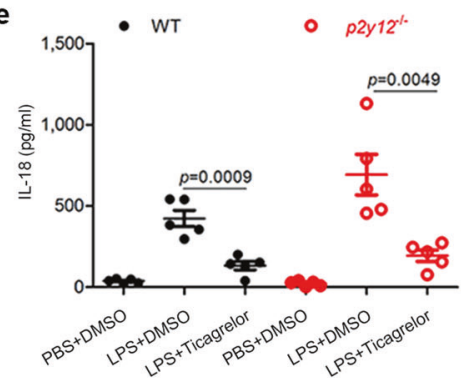

c

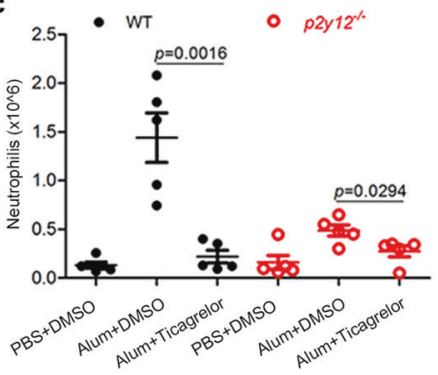

f

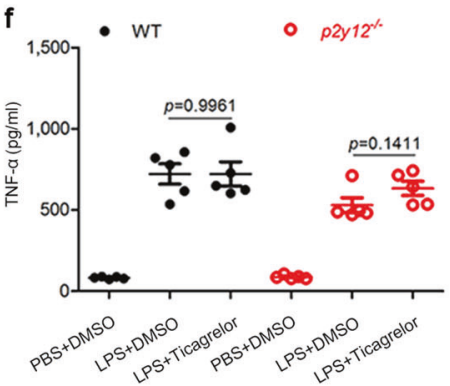

g

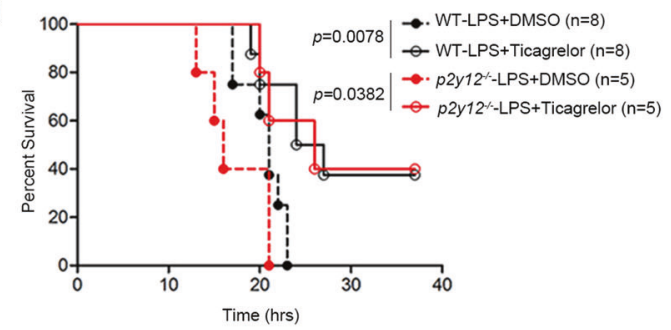

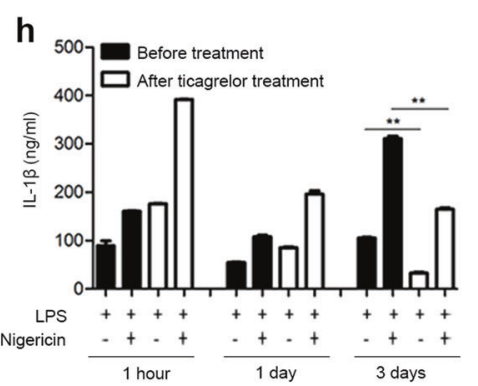

i

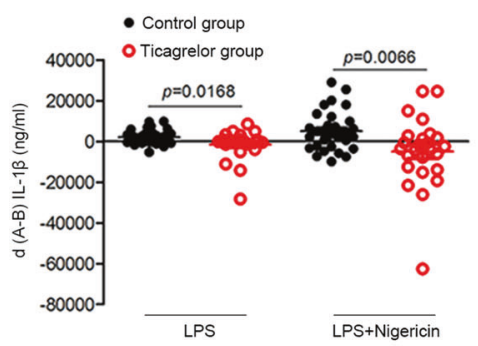

j

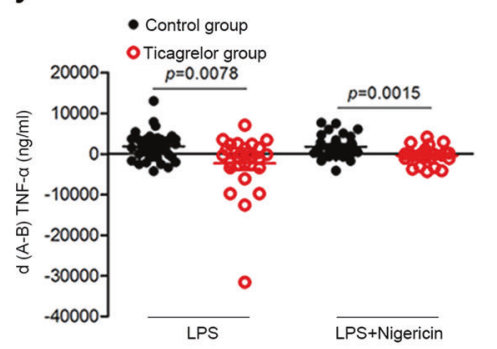

k

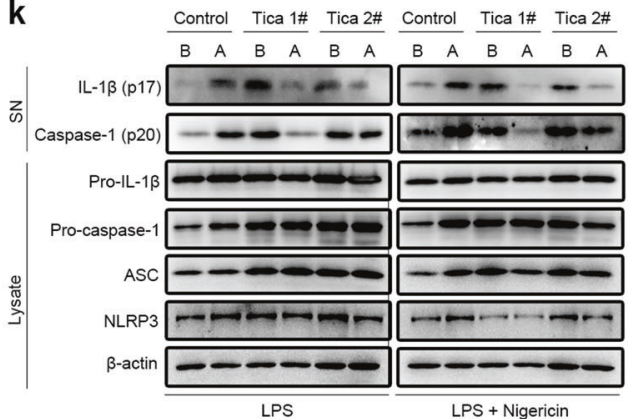

Fig. 5 Ticagrelor mitigates the severity of inflammation in vivo. a-c C57BL/6 mice (wild-type or $p 2 y 12^{-/-}$) were intraperitoneally injected with alum ( $2 \mathrm{mg} / \mathrm{mouse})$, with or without ticagrelor $(50 \mathrm{mg} / \mathrm{kg}$ body weight) and left alone for $6 \mathrm{~h}$. The peritoneal lavage fluid was collected to analyze IL-1 $\beta$ levels (a) by ELISA and the numbers of PECs (b) and neutrophils (c) by flow cytometry. d-f C57BL/6 mice (wild-type or $p 2 y 12^{-/-}$) were intraperitoneally injected with LPS $(20 \mathrm{mg} / \mathrm{kg}$ body weight) with or without ticagrelor $(50 \mathrm{mg} / \mathrm{kg}$ body weight) and left alone for $4 \mathrm{~h}$. The IL-1 $\beta$ (d), IL-18 (e), and TNF- $\alpha$ levels (f) in the serum were analyzed by ELISA. g The survival of C57BL/6 mice (wild-type or $p 2 y 12^{-1-}$ ) intraperitoneally injected with LPS ( $25 \mathrm{mg} / \mathrm{kg}$ body weight) with or without ticagrelor $(50 \mathrm{mg} / \mathrm{kg}$ body weight) was monitored. $\mathbf{h}$ PBMCs were collected from 3 patients with ACS before treatment and again after $1 \mathrm{~h}, 1$ day, or 3 days of treatment with ticagrelor. The PBMCs were primed with LPS and then stimulated with or without nigericin. Supernatants were analyzed by ELISA to determine IL-1 $\beta$ levels. $\mathbf{i}$, $\mathbf{j}$ PBMCs were collected from patients with ACS, primed with LPS, and stimulated with or without nigericin. Supernatants were analyzed by ELISA to determine IL-1 $\beta$ (i) and TNF- $\alpha$ levels (j). The difference in the level of each cytokine is termed $d(\mathrm{~A}-\mathrm{B}) ; \mathrm{A}$, after treatment; B, before treatment. Control group, $n=33$; ticagrelor group, $n=25$. k LPS-primed PBMCs from patients with ACS with or without nigericin stimulation, supernatants (SN), and cell lysates (Lysate) were analyzed by immunoblotting. Data are presented as the mean \pm SEM; unpaired, two-tailed $t-$ test; survival rates were analyzed by the Kaplan-Meier method and compared by the log-rank test. ${ }^{*} P<0.05,{ }^{* *} P<0.01$, ${ }^{* * *} P<0.001$, ns: $P>$ 0.05

with ACS after 3 days of treatment (Fig. 5h). Second, we collected PBMCs from patients with ACS before and after 3 days with or without ticagrelor treatment. There were no significant differences in clinical characteristics between the control groups (ages, 68.21 \pm 9.63 ; male/female, 14/19) and ticagrelor groups (ages, $68.04 \pm$
8.00; male/female, 15/10) (Supplemental Table 1). As shown in Supplemental Table 2, the patients with ACS in the control and ticagrelor groups took common therapeutic drugs except for ticagrelor and clopidogrel. Importantly, the most common complications of patients in the study were NLRP3-associated 


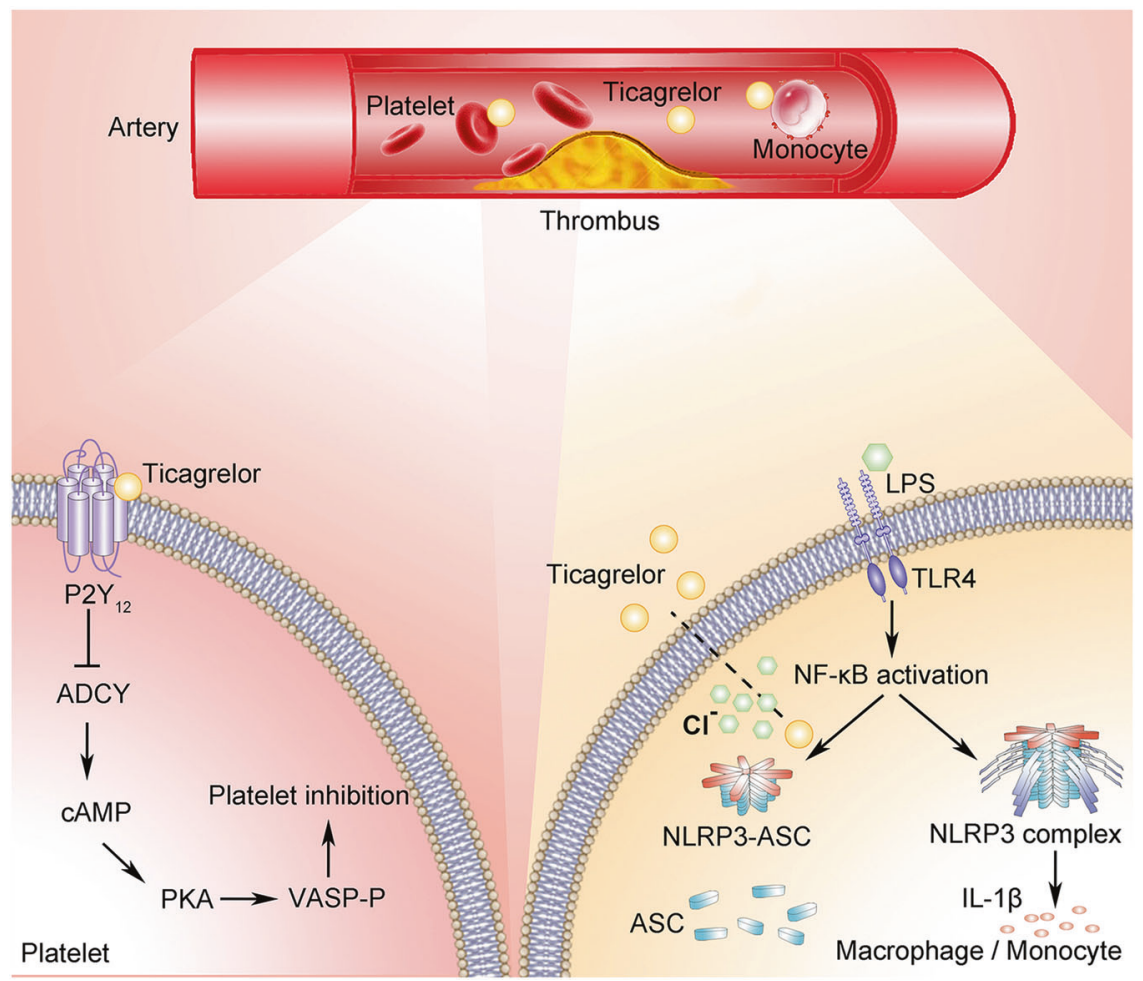

Fig. 6 The proposed model showing the different actions of ticagrelor in macrophages/monocytes and platelets. Ticagrelor controls NLRP3 inflammasome activation independent of the $\mathrm{P}_{2} \mathrm{Y}_{12}$ receptor, inhibiting ASC oligomerization by blocking chloride efflux to disrupt the formation of the inflammasome; this activity differs from the process of inhibiting platelet activation, in which ticagrelor binds to the $\mathrm{P} 2 \mathrm{Y}_{12}$ receptor on platelets, inhibiting the intracellular signaling pathway

diseases, such as hypertension, fatty liver, and diabetes (Supplemental Table 3). After treatment, the relative mRNA levels of $I I 1 \beta$, Tnfa, Nlrp3, and Asc in LPS-primed PBMCs were unchanged in the control and ticagrelor groups (Supplemental Fig. 8d-g). We then treated LPS-primed PBMCs with or without nigericin to activate the NLRP3 inflammasome and calculated the differences in inflammatory cytokine levels before and after treatment, termed $d(\mathrm{~A}-\mathrm{B})$, with a smaller $d(\mathrm{~A}-\mathrm{B})$ indicating a more potent inhibitory effect on inflammation. Our results showed that ticagrelor markedly reduced IL-1 $\beta$ and TNF- $\alpha$ production in LPSprimed PBMCs with or without nigericin stimulation compared with control treatment (Fig. $5 \mathrm{i}, \mathrm{j}$ ). Consistent with this finding, ticagrelor also blocked IL-1 $\beta$ and caspase- 1 maturation in PBMCs (Fig. 5k). These results demonstrate that ticagrelor rapidly mitigates the severity of inflammation in ACS patients in addition to inhibiting platelet activation, which demonstrates a therapeutic potential for use in NLRP3-associated diseases.

\section{DISCUSSION}

Inflammation plays a key role in the pathogenesis of CAD, which also contributes to mortality in cardiovascular disease patients. In the development of atherosclerosis, the inflamed intima produces macrophage colony-stimulating factor, and the differentiation of monocytes into macrophages leads to the release of inflammatory cytokines, chemokines proteases, etc. to cause tissue damage. ${ }^{49}$ As the best-characterized inflammasome, the NLRP3 inflammasome mediates the secretion of IL-1 $1 \beta$ and other inflammatory cytokines to initiate and promote atherosclerosis, the main cause of CAD. ${ }^{11}$ Therapeutic targeting of the NLRP3 inflammasome may benefit patients with CAD or other NLRP3associated diseases. Although extensive research has reported numerous inhibitors of the NLRP3 inflammasome, an approved medication targeting NLRP3 is not currently available. Expanding our knowledge on the inhibitory effects of various clinical medications on the NLRP3 inflammasome would facilitate therapeutic targeting of the NLRP3 inflammasome. The results from our study indicate that the widely used antiplatelet agent ticagrelor works as a potent inhibitor of the NLRP3 inflammasome. It is known that the plasma concentration of ticagrelor ranges from 0 to $2 \mu \mathrm{M},{ }^{50}$ and researchers usually use $1-10 \mu \mathrm{M}$ ticagrelor to investigate the mechanisms by which ticagrelor inhibits platelet activation. ${ }^{32}$ Inhibition of the NLRP3 inflammasome is another pharmacological role of ticagrelor distinct from its antiplatelet function. For reasons related to the specific cell type studied, the concentration of ticagrelor used in our in vitro macrophage experiments $(10-20 \mu \mathrm{M})$ was higher than the plasma concentration. Even though the plasma concentration of ticagrelor could not reach $10 \mu \mathrm{M}$ in patients, our in vitro data illustrate the potential anti-inflammatory effect of ticagrelor. Furthermore, treatment with ticagrelor inhibits activation of the NLRP3 inflammasome in the PBMCs of ACS patients, thus providing evidence for repurposing this drug for clinical application in NLRP3 inflammasome-related diseases, such as CAD.

Ticagrelor binds to the $\mathrm{P}_{2} \mathrm{Y}_{12}$ receptor, resulting in inhibition of platelet activation and aggregation by regulating the levels of CAMP and VASP-P in platelets. ${ }^{1}$ Research has also shown that macrophage inflammation is critical in the development of atherosclerosis. ${ }^{49}$ Whether ticagrelor exerts an inhibitory effect on macrophage inflammation through the $\mathrm{P}_{2} \mathrm{Y}_{12}$ receptor remains elusive. In this study, our in vitro and in vivo data demonstrate for the first time that ticagrelor inhibits activation of the NLRP3 inflammasome independent of the $\mathrm{P}_{2} \mathrm{Y}_{12}$ receptor. Our data suggest that ASC nucleation-induced oligomerization is blocked by ticagrelor without affecting the upstream step, the interaction of NLRP3 and ASC. CLIC-dependent chloride efflux participates in a variety of biological functions, which also regulate dynamic 
ASC-speck formation to activate inflammasomes. ${ }^{43}$ Pharmacological and genetic approaches both suggest that CLIC-dependent chloride efflux may serve as a potential target to treat NLRP3related diseases. ${ }^{41,51}$ Our study demonstrates that ticagrelor exerts dual functions to regulate chloride efflux in the inhibition of the NLRP3 inflammasome. First, ticagrelor induces CLIC degradation in an autophagy-dependent manner, and then it suppresses the localization of CLICs to the plasma membrane, even if CLICs are not degraded. Thus, the high levels of intracellular chloride ions induced by ticagrelor block ASC oligomerization. Notably, ASC is critical for the maturation of IL-1 $\beta$, which is released by the NLRP3, AIM2, and NLRC4 inflammasomes. ${ }^{11,12,52}$ However, ticagrelor has no effect on the activation of the NLRC4 inflammasome, which needs to be further investigated.

It is well known that a congenital deficiency in the $\mathrm{P} 2 \mathrm{Y}_{12}$ receptor and a gene polymorphism may contribute to the off-target effects of $\mathrm{P} 2 \mathrm{Y}_{12}$ antagonists, leading to serious consequences in patients. ${ }^{53}$ Interestingly, our studies in mouse models demonstrate that ticagrelor inhibits NLRP3 inflammasome activation in vivo to protect against inflammatory disease independent of its classic inhibitory effect on the $\mathrm{P}_{2} \mathrm{Y}_{12}$ signaling pathway. The inhibitory effect of ticagrelor on the NLRP3 inflammasome may also benefit patients with defects in the $\mathrm{P}_{2} \mathrm{Y}_{12}$ receptor. Importantly, our results showing that ticagrelor rapidly and significantly regulates inflammasome activation in immune cells from ACS patients highlight the potential therapeutic effect of ticagrelor on NLRP3-associated diseases. The ACS patients enrolled in our study suffered from multiple NLRP3-associated diseases, such as hypertension, fatty liver, and diabetes. These complex diseases and therapeutic drugs to treat them may affect the inflammatory response and immune functions of patients. However, PBMCs obtained from patients with ACS after 3 days of oral treatment including ticagrelor showed lower levels of inflammatory cytokines in response to inflammasome stimuli than PBMCs from patients without ticagrelor treatment, indicating that ticagrelor plays an anti-inflammatory role in ACS patients. A randomized, double-blinded clinical trial is needed to investigate the potential therapeutic effects of ticagrelor on NLRP3-associated diseases.

Overall, our work demonstrates a novel pharmacological function of ticagrelor independent of its classic effect on the $\mathrm{P}_{2 \mathrm{Y}_{12}}$ receptor: inhibition of NLRP3 inflammasome activation in macrophages. Mechanistically, as illustrated in Fig. 6, ticagrelor inhibits the oligomerization of ASC, an effect achieved through the degradation of CLICs and blockade of the translocation of CLICs to the plasma membrane, thus blocking chloride efflux. More importantly, the results from in vivo mouse models and ACS patients support the clinical application of ticagrelor in NLRP3associated diseases.

\section{MATERIAL AND METHODS}

Mice

All animal experiments were approved by the Review Committee of Zhejiang University School of Medicine and were in compliance with institutional guidelines. All mice were kept and bred in-house at the laboratory animal center of Zhejiang University. Experiments were carried out in 6- to 8-week-old male mice. All mice were on the C57BL/6 background (wild-type mice) for the in vitro experiments and were purchased from Shanghai SLAC Laboratory Animal Co., Ltd., and $p 2 y 12^{-1-}$ mice 28 were obtained from Professor Junling Liu, Shanghai Jiao Tong University.

\section{Human participants}

The protocol for this research was approved by the Ethics Committee of Hangzhou First People's Hospital, and the experimental protocol was performed in accordance with the Declaration of Helsinki. Written informed consent to collect samples and participate in the study was obtained from all patients. Acute coronary syndrome patients were enrolled according to the diagnostic criteria of the American Heart Association. Fasting venous blood was collected from patients in an ethylenediaminetetraacetic acid-anticoagulant tube the morning after they were hospitalized (day 0). Patients received routine medical care without specific interventions. The patients in the ticagrelor group were treated with $90 \mathrm{mg}$ ticagrelor (AstraZeneca, London, UK) twice daily from day 0 to day 2, and the patients in the control group did not receive ticagrelor. After 3 days, we collected fasting peripheral venous blood again. PBMCs were isolated from peripheral venous blood for further experiments.

\section{Cell cultures and stimulation}

Bone marrow cells isolated from the tibia and femur of C57BL/6 wild-type mice or $p 2 y 12^{-1-}$ mice were differentiated for 5 days in Dulbecco's modified Eagle medium (DMEM) supplemented with $10 \%$ fetal bovine serum, $1 \%$ penicillin/streptomycin, and $10 \mathrm{ng} / \mathrm{ml}$ murine macrophage colony-stimulating factor (PeproTech, Rocky Hill, NJ, USA) to generate bone marrow-derived macrophages (BMDMs). Mice were injected intraperitoneally with $1 \mathrm{ml} 3 \%$ fluid thioglycollate medium (wt/vol) (Merck, Darmstadt, Germany) for 5 days to obtain peritoneal macrophages (PMs). BMDMs or PMs were seeded at $6 \times 10^{5} / \mathrm{ml}$ in 12-well plates overnight, and the medium was changed to Opti-MEM (Invitrogen, Carlsbad, CA, USA) containing $500 \mathrm{ng} / \mathrm{ml}$ LPS (Sigma, St. Louis, MO, USA) for $6 \mathrm{~h}$. Next, the cells were stimulated with an NLRP3 inflammasome activator, $10 \mu \mathrm{M}$ nigericin (InvivoGen, San Diego, CA, USA) for $30 \mathrm{~min}$, $2.5 \mathrm{mM}$ ATP (Sigma, St. Louis, MO, USA) for $30 \mathrm{~min}, 320 \mu \mathrm{g} / \mathrm{ml}$ alum (Thermo Fisher Scientific, Waltham, MA, USA) for $4 \mathrm{~h}$, or $300 \mu \mathrm{g} / \mathrm{ml}$ MSU (InvivoGen, San Diego, CA, USA) for $4 \mathrm{~h}$. Cells were transfected with $1 \mu \mathrm{g} / \mathrm{ml}$ poly (dA:dT) using Lipofectamine 2000 (Invitrogen, Carlsbad, CA, USA) for $30 \mathrm{~min}$ to activate the AIM2 inflammasome. For NLRC4 inflammasome activation, after 15 min of Salmonella infection, cells were incubated with gentamycin for another 30 min. Ticagrelor, ZM241385, MG132, and Bafilomycin A1 were obtained from Selleck Chemicals (Houston, TX, USA) and dissolved in dimethyl sulfoxide (DMSO) or $\mathrm{ddH}_{2} \mathrm{O}$. The final dilution of DMSO or $\mathrm{dd}_{2} \mathrm{O}$ was $\leq 1: 1,000$. Supernatants were collected and analyzed using ELISA kits according to the manufacturers' instructions. Cell lysates and precipitated supernatants were analyzed by Western blotting.

\section{ELISA}

Supernatants from cell culture, serum, and peritoneal cavity fluid samples were used to determine the levels of IL-1 $\beta, I L-18$, and TNF-a (eBioscience, San Diego, CA, USA), and cell lysates were analyzed for CAMP levels according to the manufacturers' instructions.

\section{Western blotting}

After protein separation, transfer and blocking, membranes were incubated with antibodies. The primary antibodies used for mouse and human experiments were anti-IL-1 $\beta$ (12242) (1:1,000; Cell Signaling Technology, Danvers, MA, USA), anti-NLRP3 (AG-20B0014) (1:1,000; AdipoGen, San Diego, CA, USA), anti-ASC (AG-25B0006) (1:1,000; AdipoGen, San Diego, CA, USA), and anti- $\beta$-actin (KM9001) (1:5,000; Sungene Biotech, Tianjin, China). The antibodies against mouse antigens were anti-Ub (sc-8017) (1:1,000; Santa Cruz, Santa Cruz, CA, USA), anti-caspase-1 (AG-20B-0042) (1:1,000; AdipoGen, San Diego, CA, USA), anti-Clic1 (D222732) (1:1,000; BBI, Shanghai, China), anti-Clic4 (D161568) (1:1,000; BBI, Shanghai, China), anti-Clic5 (ab66630) (1:500; Abcam, Cambridge, MA, USA), anti-GAPDH (70-Mab5465-040) (1:1,000; MultiSciences, Hangzhou, China), and anti-ATP1A1 (A01483) (1:1,000; GenScript, Nanjing, China). Anti-caspase-1 (A0964) (1:1,000; Abclonal Technology, Wuhan, China) was used to detect the corresponding human protein. The secondary HRP-conjugated antibodies were anti-rabbit IgG (W401B) (1:10,000; Promega, Madison, WI, USA) 
and anti-mouse IgG (W402B) (1:10,000; Promega, Madison, WI, USA).

ASC oligomerization and ASC-speck formation

BMDMs $\left(6 \times 10^{6}\right)$ were seeded in $6-\mathrm{cm}$ dishes, primed with $500 \mathrm{ng} /$ $\mathrm{ml}$ LPS for $6 \mathrm{~h}$, and stimulated with $10 \mu \mathrm{M}$ nigericin for $30 \mathrm{~min}$. Then, the culture medium was removed, and the cells were washed with cold phosphate-buffered saline (PBS). Cold buffer $(500 \mu \mathrm{l}$ of $50 \mathrm{mM}$ Tris- $\mathrm{HCl}, 0.5 \%$ Triton X-100, $1 \mathrm{mM}$ dithiothreitol, and $1 \mathrm{mM}$ phenylmethylsulfonyl fluoride in NP-40 lysis buffer) was added to the cells. The cells were transferred to Eppendorf tubes and pipetted 20 times with a 21 -gauge needle. Next, the tubes were centrifuged at $330 \times \mathrm{g}$ at $4^{\circ} \mathrm{C}$ for $10 \mathrm{~min}$, and the precipitate was collected and washed twice with cold PBS. The precipitate was resuspended in $500 \mu \mathrm{l}$ cold PBS containing $4 \mathrm{mM}$ Suberic acid bis (N-hydroxysuccinimide ester) (Sigma, St. Louis, MO, USA) and then incubated with rotation at room temperature for $30 \mathrm{~min}$. The samples were centrifuged to obtain precipitates for Western blotting. For ASC-speck formation, 10-mm cell culture slides were placed in the bottom of 6 -well plates. BMDMs were plated at $6 \times$ $10^{5} / \mathrm{ml}$ in the 6 -well plates for analysis of ASC-speck formation, which represented ASC oligomerization. The cells were fixed in 4\% paraformaldehyde and incubated with an anti-ASC antibody (1:50), a DyLight488-conjugated anti-rabbit IgG antibody (1:100; MultiSciences, Hangzhou, China) and DAPI $(2.5 \mu \mathrm{g} / \mathrm{ml}$; Beyotime Biotechnology, Shanghai, China). ASC-speck formation was imaged under a positive fluorescence microscope (Nikon, Tokyo, Japan).

Gene silencing and editing in BMDMs

BMDMs were plated at $6 \times 10^{5} / \mathrm{ml}$ in 12 -well plates and then transfected with 60 pmol siRNA using Lipofectamine 2000 (Invitrogen, Carlsbad, CA, USA) for $6 \mathrm{~h}$ to silence $p 2 y 12$. After that, the siRNA-containing medium was changed to DMEM for another $18 \mathrm{~h}$. The siRNAs targeting p2y12 were 5'-GCCAAGUUACUUCAGUCA CAUTT- $3^{\prime}$ and $5^{\prime}$-GCACGAAAUAGUCAAUUACAUTT- $3^{\prime}$, the siRNAs targeting Syk were 5'-CCAUCGAGAGGGAACUUAATT-3' and $5^{\prime}$ GGAUCAAAUCCUACUCCUUTT- $3^{\prime}$, and the negative control siRNA was $5^{\prime}$-UUCUCCGAACGUGUCACGUTT-3'. The relative mRNA level of $p 2 y 12$ was analyzed by quantitative real-time PCR. CRISPR/Cas9 was applied to knock out JNK1/2 in immortal BMDMs (iBMDMs).

Identification of $p 2 y 12^{-1-}$ mice

One to two millimeters of mouse tail tissue was heated in $400 \mu \mathrm{l}$ $50 \mathrm{mM} \mathrm{NaOH}$ for $20 \mathrm{~min}$ at $100^{\circ} \mathrm{C}$, and $40 \mu \mathrm{l}$ Tris- $\mathrm{HCl}(0.5 \mathrm{M}, \mathrm{pH}$ 6.8) was added to the supernatant to prepare template DNA. One

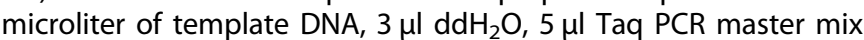
(Sangon Biotech, Shanghai, China), and $1 \mu$ l primer were mixed for PCR. The forward primer was 5'-GGCTGCCTTGAGAAATATCAAGT$3^{\prime}$, the reverse primer for wild-type mice was $5^{\prime}$-GGCACTCTAGT GATGCTITGCCTA- $3^{\prime}$, and the reverse primer for $p 2 y 12^{-1-}$ mice was $5^{\prime}$-ACGCGTCACCTTAATATGC- $3^{\prime}$. The PCR products were resolved by $1.5 \%$ agarose gel electrophoresis and visualized with an ultraviolet transilluminator.

PCR and quantitative real-time PCR

Relative mRNA levels were determined using gene-specific primers. The gene-specific primers for BMDMs or PBMCs were as follows: mouse-p2y12 1\#, 5'-CATTGCTGTACACCGTCCTG-3' and 5'GGCTCCCAGTITAGCATCAC-3'; mouse-p2y12 2\#, 5'-CACGGATTC CCTACACCCTG- $3^{\prime}$ and $5^{\prime}$-GGGTGCTCTCCTTCACGTA G-3'; mouse- $\beta$ actin, $5^{\prime}$-CGTTGACATCCGTAAAGACC- $3^{\prime}$ and $5^{\prime}$-AACAGTCCGCCTA GAAGCAC-3'; human- $I L-1 \beta, 5^{\prime}$-GGATATGGAGCAACAAGTGG-3' and 5'-ATGTACCAGTTGGGGAACTG-3'; human-TNF- $a$, 5'-CAGAGGGAA GAGTTCCCCAG-3' and 5'-CCTTGGTCTGGTAGGAGACG-3'; humanNLRP3， 5'-ATGCCAGGAAGACAGCATTG-3' and 5'-TCATCGAAGC CGTCCATGAG-3'; human-ASC, 5'-AACCCAAGCAAGATGCGGAA G-3' and 5'-TTAGGGCCTGGAGGAGCAAG-3'; and human- $\beta$-actin,
5'-CTGGGACGACATGGAGAAAA-3' and 5'-AAGGAAGGCTGGAAG AGTGC-3'

Determination of the intracellular chloride ion level

To detect intracellular chloride ions, supernatants were removed before washing lysates with PBS. BMDMs were incubated with 5 mM MQAE (Beyotime Biotechnology, Shanghai, China) for $1 \mathrm{~h}$. After washing the lysates with PBS three times, the intracellular fluorescence intensity of MQAE was detected by fluorescence microscopy. A higher fluorescence intensity of MQAE indicates a lower level of intracellular chloride ions and vice versa.

Isolation of the plasma membrane

To detect the translocation of CLICs to the plasma membrane, the cytosolic and plasma membrane fractions were purified with the Minute Plasma Membrane Protein Isolation and Cell Fractionation Kit (Invent Biotechnologies, Plymouth, MN, USA) according to the manufacturers' instructions.

Alum-induced peritonitis mouse model

To establish alum-induced peritonitis in vivo, C57BL/6 wild-type or p2y $12^{-1-}$ mice were injected intraperitoneally with DMSO and $2 \mathrm{mg}$ alum dissolved in $0.2 \mathrm{ml}$ sterile PBS and left alone for $6 \mathrm{~h}$. Negative control mice were injected intraperitoneally with DMSO and PBS. In the group treated with ticagrelor, the mice were injected intraperitoneally with alum and ticagrelor $(50 \mathrm{mg} / \mathrm{kg}$ body weight). After alum challenge, mice were sacrificed to analyze the activation of inflammation in vivo. The peritoneal cavity was washed with PBS, and IL-1 $\beta$ secretion was measured by ELISA. PECs were stained with the neutrophil markers Ly6G (BD Pharmingen, San Diego, CA, USA) and CD11b (BD Pharmingen, San Diego, CA, USA), and then the numbers of PECs and neutrophils were calculated using ACEA NovoCyte. Mice were allocated into experimental groups by simple randomization. After group allocation, one investigator performed the experiments on each group, and a second investigator blinded to the groups collected and analyzed the data.

LPS-induced sepsis in vivo

LPS was dissolved in PBS to obtain a stock solution before in vivo experiments. To induce cytokine secretion in vivo, C57BL/6 wildtype or $p 2 y 12^{-1-}$ mice were injected intraperitoneally with DMSO and LPS ( $20 \mathrm{mg} / \mathrm{kg}$ body weight) and left alone for $4 \mathrm{~h}$. Negative control mice were injected intraperitoneally with DMSO and PBS. In the group treated with ticagrelor, the mice were injected intraperitoneally with LPS and ticagrelor $(50 \mathrm{mg} / \mathrm{kg}$ body weight). Next, serum samples were collected. The IL-1 $\beta$, IL-18, and TNF- $a$ levels in the serum were measured using ELISA. Twenty-five milligrams/kg body weight LPS was used to evaluate percentage survival in LPS-induced sepsis. Deaths were recorded every hour. Percentage survival was analyzed using the Kaplan-Meier method, and outcomes were compared using the log-rank test. Mice were allocated into experimental groups by simple randomization. After group allocation, one investigator performed the experiments on each group, and a second investigator blinded to the groups collected and analyzed the data.

Statistical analyses

Data presented as the mean \pm SEM were compared using an unpaired, two-tailed $t$-test. Data were considered significant when $P<0.05$.

\section{ACKNOWLEDGEMENTS}

This work was supported by the National Natural Science Foundation of China (Grant Nos. 81773016, 21976155, and 31471297), Zhejiang Provincial Natural Science Foundation of China (No. LY18C060001), WeiJian Special Foundation (Zhejiang University School of Public Health), and Fundamental Research Funds for the Central 
Universities. The authors thank Professor Feng Shao (National Institute of Biological Sciences, Beijing, China) for providing iBMDMs. The authors thank Dr. Lain Charles Bruce (The University of Hong Kong) for his linguistic advice. The authors thank the Core Facilities at the Zhejiang University School of Medicine for providing technical support.

\section{AUTHOR CONTRIBUTIONS}

D.X., Y.W., B.H., Y.Q., S.X., X.Y., H.H., S.M., J.X., P.H., D.W., and H.-M.S. designed the study. B.H., Y.Q., S.X., H.C., Z.C., and L.Z. performed the experiments in this work. D.X., Y.W., B. H., and S.X. analyzed the data in this study. D.X., Y.W., and B.H. wrote the manuscript. All authors contributed to the discussion of the study and revision of the manuscript.

\section{ADDITIONAL INFORMATION}

The online version of this article (https://doi.org/10.1038/s41423-020-0444-5) contains supplementary material.

Competing interests: The authors declare no competing interests.

\section{REFERENCES}

1. Rollini, F., Franchi, F. \& Angiolillo, D. J. Switching $P 2 Y_{12}$-receptor inhibitors in patients with coronary artery disease. Nat. Rev. Cardiol. 13, 11-27 (2016).

2. Gaglia, M. A. Jr. \& Waksman, R. Overview of the 2010 Food and Drug Administration Cardiovascular and Renal Drugs Advisory Committee meeting regarding ticagrelor. Circulation 123, 451-456 (2011).

3. Wallentin, L. et al. Ticagrelor versus clopidogrel in patients with acute coronary syndromes. N. Engl. J. Med. 361, 1045-1057 (2009).

4. Alexopoulos, D. et al. Ticagrelor versus prasugrel in acute coronary syndrome patients with high on-clopidogrel platelet reactivity following percutaneous coronary intervention: a pharmacodynamic study. J. Am. Coll. Cardiol. 60, 193-199 (2012).

5. Roffi, M. et al. 2015 ESC guidelines for the management of acute coronary syndromes in patients presenting without persistent ST-segment elevation: Task Force for the Management of Acute Coronary Syndromes in patients presenting without persistent ST-segment elevation of the European Society of Cardiology (ESC). Eur. Heart J. 37, 267-315 (2016).

6. Veitch, A. M. et al. Endoscopy in patients on antiplatelet or anticoagulant therapy, including direct oral anticoagulants: British Society of Gastroenterology (BSG) and European Society of Gastrointestinal Endoscopy (ESGE) guidelines. Gut 65, 374-389 (2016).

7. Varenhorst, C. et al. Factors contributing to the lower mortality with ticagrelor compared with clopidogrel in patients undergoing coronary artery bypass surgery. J. Am. Coll. Cardiol. 60, 1623-1630 (2012).

8. Storey, R. F. et al. Lower mortality following pulmonary adverse events and sepsis with ticagrelor compared to clopidogrel in the PLATO study. Platelets 25, 517-525 (2014).

9. Jeong, H. S. et al. Comparison of ticagrelor versus prasugrel for inflammation, vascular function, and circulating endothelial progenitor cells in diabetic patients with non-ST-segment elevation acute coronary syndrome requiring coronary stenting: a prospective, randomized, crossover trial. JACC Cardiovasc. Interv. 10, 1646-1658 (2017).

10. Birnbaum, Y., Birnbaum, G. D., Birnbaum, I., Nylander, S. \& Ye, Y. Ticagrelor and rosuvastatin have additive cardioprotective effects via adenosine. Cardiovasc. Drugs Ther. 30, 539-550 (2016).

11. Guo, H., Callaway, J. B. \& Ting, J. P. Inflammasomes: mechanism of action, role in disease, and therapeutics. Nat. Med. 21, 677-687 (2015).

12. Lamkanfi, M. \& Dixit, V. M. Mechanisms and functions of inflammasomes. Cell 157, 1013-1022 (2014).

13. Swanson, K. V., Deng, M. \& Ting, J. P. The NLRP3 inflammasome: molecular activation and regulation to therapeutics. Nat. Rev. Immunol. 19, 477-489 (2019).

14. Schroder, K. \& Tschopp, J. The inflammasomes. Cell 140, 821-832 (2010).

15. Chen, G. Y. \& Nunez, G. Sterile inflammation: sensing and reacting to damage. Nat. Rev. Immunol. 10, 826-837 (2010).

16. Robbins, G. R., Wen, H. \& Ting, J. P. Inflammasomes and metabolic disorders: old genes in modern diseases. Mol. Cell 54, 297-308 (2014).

17. Toldo, S. \& Abbate, A. The NLRP3 inflammasome in acute myocardial infarction. Nat. Rev. Cardiol. 15, 203-214 (2016).

18. Unamuno, $X$. et al. NLRP3 inflammasome blockade reduces adipose tissue inflammation and extracellular matrix remodeling. Cell. Mol. Immunol. https://doi. org/10.1038/s41423-019-0296-z (2019).
19. Coll, R. C. et al. A small-molecule inhibitor of the NLRP3 inflammasome for the treatment of inflammatory diseases. Nat. Med. 21, 248-255 (2015).

20. Jiang, $H$. et al. Identification of a selective and direct NLRP3 inhibitor to treat inflammatory disorders. J. Exp. Med. 214, 3219-3238 (2017).

21. Yan, Y. et al. Dopamine controls systemic inflammation through inhibition of NLRP3 inflammasome. Cell 160, 62-73 (2015).

22. Guo, C. et al. Bile acids control inflammation and metabolic disorder through inhibition of NLRP3 inflammasome. Immunity 45, 802-816 (2016).

23. Horng, T. Calcium signaling and mitochondrial destabilization in the triggering of the NLRP3 inflammasome. Trends Immunol. 35, 253-261 (2014).

24. Davis, B. K., Wen, H. \& Ting, J. P. The inflammasome NLRs in immunity, inflammation, and associated diseases. Annu. Rev. Immunol. 29, 707-735 (2011).

25. Hornung, V. et al. AIM2 recognizes cytosolic dsDNA and forms a caspase-1activating inflammasome with ASC. Nature 458, 514-518 (2009).

26. Vance, R. E. The NAIP/NLRC4 inflammasomes. Curr. Opin. Immunol. 32, 84-89 (2015).

27. Kronlage, M. et al. Autocrine purinergic receptor signaling is essential for macrophage chemotaxis. Sci. Signal. 3, ra55 (2010).

28. $\mathrm{Li}, \mathrm{D}$. et al. Roles of purinergic receptor P2Y, G protein-coupled 12 in the development of atherosclerosis in apolipoprotein E-deficient mice. Arterioscler. Thromb. Vasc. Biol. 32, e81-e89 (2012).

29. Daniel, J. L. et al. Molecular basis for ADP-induced platelet activation. I. Evidence for three distinct ADP receptors on human platelets. J. Biol. Chem. 273, 2024-2029 (1998).

30. Hollopeter, G. et al. Identification of the platelet ADP receptor targeted by antithrombotic drugs. Nature 409, 202-207 (2001).

31. Foster, C. J. et al. Molecular identification and characterization of the platelet ADP receptor targeted by thienopyridine antithrombotic drugs. J. Clin. Investig. 107, 1591-1598 (2001)

32. Aungraheeta, R. et al. Inverse agonism at the P2Y12 receptor and ENT1 transporter blockade contribute to platelet inhibition by ticagrelor. Blood 128, 2717-2728 (2016)

33. Lee, G. S. et al. The calcium-sensing receptor regulates the NLRP3 inflammasome through $\mathrm{Ca}^{2+}$ and cAMP. Nature 492, 123-127 (2012).

34. Bauernfeind, F. G. et al. Cutting edge: NF-kappaB activating pattern recognition and cytokine receptors license NLRP3 inflammasome activation by regulating NLRP3 expression. J. Immunol. 183, 787-791 (2009).

35. Py, B. F., Kim, M. S., Vakifahmetoglu-Norberg, H. \& Yuan, J. Deubiquitination of NLRP3 by BRCC3 critically regulates inflammasome activity. Mol. Cell 49, 331-338 (2013).

36. Juliana, C. et al. Non-transcriptional priming and deubiquitination regulate NLRP3 inflammasome activation. J. Biol. Chem. 287, 36617-36622 (2012).

37. Lu, A. et al. Unified polymerization mechanism for the assembly of ASCdependent inflammasomes. Cell 156, 1193-1206 (2014).

38. Cai, X. et al. Prion-like polymerization underlies signal transduction in antiviral immune defense and inflammasome activation. Cell 156, 1207-1222 (2014).

39. Vajjhala, P. R., Mirams, R. E. \& Hill, J. M. Multiple binding sites on the pyrin domain of ASC protein allow self-association and interaction with NLRP3 protein. J. Biol. Chem. 287, 41732-41743 (2012).

40. Hara, $\mathrm{H}$. et al. Phosphorylation of the adaptor ASC acts as a molecular switch that controls the formation of speck-like aggregates and inflammasome activity. Nat. Immunol. 14, 1247-1255 (2013).

41. Tang, T. et al. CLICs-dependent chloride efflux is an essential and proximal upstream event for NLRP3 inflammasome activation. Nat. Commun. 8, 202 (2017).

42. Domingo-Fernandez, R., Coll, R. C., Kearney, J., Breit, S. \& O'Neill, L. A. J. The intracellular chloride channel proteins CLIC1 and CLIC4 induce IL-1beta transcription and activate the NLRP3 inflammasome. J. Biol. Chem. 292, 12077-12087 (2017).

43. Green, J. P. et al. Chloride regulates dynamic NLRP3-dependent ASC oligomerization and inflammasome priming. Proc. Natl. Acad. Sci. U.S.A. 115, E9371-E9380 (2018).

44. Park, S. H., Hyun, J. Y. \& Shin, I. A lysosomal chloride ion-selective fluorescent probe for biological applications. Chem. Sci. 10, 56-66 (2019).

45. Littler, D. R. et al. The enigma of the CLIC proteins: lon channels, redox proteins, enzymes, scaffolding proteins? FEBS Lett. 584, 2093-2101 (2010).

46. Li, H., Willingham, S. B., Ting, J. P. Y. \& Re, F. Cutting edge: inflammasome activation by alum and alum's adjuvant effect are mediated by NLRP3. J. Immunol. 181, 17-21 (2008)

47. Sutterwala, F. S. et al. Critical role for NALP3/CIAS1/Cryopyrin in innate and adaptive immunity through its regulation of caspase-1. Immunity 24, 317-327 (2006).

48. Steg, P. G. et al. Ticagrelor versus clopidogrel in patients with ST-elevation acute coronary syndromes intended for reperfusion with primary percutaneous 
Ticagrelor inhibits the NLRP3 inflammasome to protect against...

B Huang et al.

coronary intervention: a Platelet Inhibition and Patient Outcomes (PLATO) trial subgroup analysis. Circulation 122, 2131-2141 (2010).

49. Hansson, G. K. Inflammation, atherosclerosis, and coronary artery disease. N. Engl. J. Med. 352, 1685-1695 (2005).

50. McEvoy, J. W. et al. Effect of intravenous fentanyl on ticagrelor absorption and platelet inhibition among patients undergoing percutaneous coronary intervention: the PACIFY Randomized Clinical Trial (platelet aggregation with ticagrelor inhibition and fentanyl). Circulation 137, 307-309 (2018).
51. Daniels, M. J. et al. Fenamate NSAIDs inhibit the NLRP3 inflammasome and protect against Alzheimer's disease in rodent models. Nat. Commun. 7, 12504 (2016).

52. Broz, P. et al. Redundant roles for inflammasome receptors NLRP3 and NLRC4 in host defense against Salmonella. J. Exp. Med. 207, 1745-1755 (2010).

53. Cattaneo, M. The platelet P2Y(1)(2) receptor for adenosine diphosphate: congenital and drug-induced defects. Blood 117, 2102-2112 (2011). 